\title{
Farewell and recognitions from the Editor in Chief
}

\author{
Jos A. E. Spaan
}

Received: 15 November 2012/ Accepted: 16 November 2012

(C) International Federation for Medical and Biological Engineering 2012

It was a privilege to serve Medical and Biological Engineering and Computing, the official journal of the International Federation of Medical and Biological Engineering, IFMBE. When the new Editorial team took over in July 2005 from Alan Murray, many changes were implemented. The most important one was the introduction of Web-based handling of the manuscripts. This allowed a faster response from authors and much better communication between editor and his associates as well as the reviewers.

One of the aims of the new editorial team was to improve the Impact Factor of the journal but not at the expense of indices that were already good such as the cited half life which was and remained close to 10 years [1]. The impact factor went up from about 1 to 1.89 , a substantial increase indeed. It makes no sense to speculate on why it did not increase further. What did really happen was a rapid doubling of the number of submissions over this editorial period. It demonstrates the pressure we are all experiencing to publish. However, the quality of the increased paper flow is not always as high as previously. I would like to thank whole heartedly the reviewers who worked so hard for the journal. The increased number of papers did put a high demand on reviewers. We implemented editorial triage to reduce the amount of review requests and to increase the quality of papers that went out for review. We have listed the 103 reviewers who wrote one or more review per year in average over the last 6 years in Appendix Table 1. Those 559 reviewers who helped us out in 2012 are listed in Appendix Table 2.

The editorial board should be recognized as well. We have used the services of these members in case there was

\section{J. A. E. Spaan $(\square)$}

Department of Biomedical Engineering,

Academic Medical Center, University of Amsterdam,

Amsterdam, The Netherlands

e-mail: j.a.spaan@amc.uva.nl uncertainty in the review process and critical advice was needed.

I like to express gratitude towards the associate editors. We started out with Ruben Coronel, Maria Siebes, Jenny Dankelman and Ed van Bavel. It was my choice to have this first team geographically close as to optimize communication. Nico Schellart joined somewhat later. After the first 3 years, Jenny and Ed left the team and were replaced by Dario Farina from Denmark and Andreas Wahle from Iowa, USA. All these associate editors had their specialty and took part of the editorial load. However, I like to thank especially Ruben Coronel for his support. Ruben is a born editor with a very mature judgment and a high efficiency in decision making. His collegiality was always refreshing which became also clear when the delay in nomination of a new Editor in Chief and the steady increase in submission resulted in too high a burden for the Editor in Chief. Ruben took care of all new submissions since Sept 12012.

The IFMBE owns the journal and considers it its Flagship. Although the direct responsibility of the journal is with the Federal Journal Committee, FJC, the four presidents of the IFMBE who were involved in my editorial period showed all direct interest in the journal be it in different intensity. Joachim Nagel, Makoto Kikuchi, Herb Voigt and since recently Ratko Magjarević. Joachim did put in a tremendous effort in realizing the journal transition but I would like to recognize especially Makoto Kikuchi and Herb Voigt for their understanding and support for Editorial affairs.

To me, it is very clear that the Founding Fathers of the IFMBE were wise in having the rules of engagement between IFMBE and Editors spelled out in the constitution. The FJC has been constructed as a committee with own responsibility towards the journal. In my opinion, this 
should remain so and I thank David Holder whole heartedly for his support during my editorship. There have been people in the background giving me tremendous support. Just two, I would like to mention are Tobias Op ' $t$ Hof and Krishnan Shankar. Tobias has great insight in how journals operate and how mechanisms of citation do affect the quality ratings discussed elsewhere in this issue. Krishnan was not only the treasurer of the IFMBE but a true friend who kept in an informal way everyone connected. Springer was a great help over this period. It was a pleasure to work with of all of its representatives. Three I like to name explicitly. Dieter Merkle was our liaison manager within Springer. He must have been amazed in the beginning about those strong character scientists involved in the journal but he did a beautiful job in providing us with professional guidance. At the beginning of the second contract period, his task was taken over by Christoph Baumann who again was very professional and helpful. Claudia Kehl at Springer was our journal manager at Springer in the first and the last years of my editorship. She was always understanding. In the first years for the slow flow of papers and the last few months for a slow Editor in Chief.

Last but not least, I like to thank the authors who submitted their work to Medical and Biological Engineering and Computing. We published together about 700 papers which means that one way or the other we communicated and resolved our issues with the manuscripts when ever present. In the end, they are the authors who make the journal. Especially, there are many young authors I wish well for their careers and I hope they will continue with finding pleasure in their work and many more papers to come.

\section{Appendix}

See Appendix for Tables 1 and 2 .

Table 1 Reviewers (103) with more than 6 review reports over the last 7 year

Abächerli, Roger (6)

Acharya u, Rajendra (8)

Al-Ani, Tarik (7)

Antolini, Renzo (11)

Arslan, Yunus Ziya (8)

Arts, Theo (6)

Audenaert, Emmanuel (7)
Table 1 continued

Avolio, Alberto (19)

Aydin, Serap (6)

Bakker, Erik (6)

Baumert, Mathias (8)

Blanco, Susana (8)

Bour, Lo (6)

Campo, Oscar (7)

Canton, Gador (8)

Cappello, Angelo (6)

Carus, David (6)

Casaleggio, Aldo (8)

Chen, Jia-Jin (8)

Cherng, Shen (7)

Chien, Aichi (7)

Chui, Cheekong (12)

Coronel, Ruben (25)

Cretual, Armel (6)

Cutti, Andrea Giovanni (14)

Cysarz, D. (6)

Dankelman, Jenny (9)

de Bakker, Jacques (9)

Dotsinsky, Ivan Assenov (16)

Farina, Dario (17)

Fenster, Aaron (7)

Folland, Ross (7)

Fuentes Cabrera, Alvaro (7)

Gefen, Amit (15)

Gianfelici, Francesco (6)

Grill, Warren M. (6)

Grimbergen, C. A. (21)

Guler, Inan (7)

Hadjileontiadis, L. J. (7)

Holder, David (6)

Hund, Thomas (8)

Jazbinsek, Vojko (10)

Jiang, Ning (8)

Kochina, Marina (6)

Konings, Maurits K. (6)

Krams, Rob (6)

Kulkas, Antti Eemeli (6)

Kwok, Yeeli (17)

Lackovic, Igor (6)

Laguna, Pablo (7)

Lee, Kyoung-Joung (6)

Liberati, Diego E.I. (13)

Linnenbank, Andre (12)

Lujan, J. Luis (14)

Madeleine, Pascal (9)

Marcos, J. Víctor (6) 
Table 1 continued

Mason, David Glen (9)

Matsopoulos, George K. (11)

McGill, Kevin (6)

Mendez, Martin Oswaldo (11)

Molinari, Filippo (16)

Moussavi, Zahra (8)

Noshiro, Makoto (7)

Op 't Hof, Tobias (10)

Orizio, Claudio (8)

Panerai, Ronney (6)

Papaharilaou, Yannis (10)

Parker, Kim (7)

Patil, Mothiram Keelanje (8)

Penzel, Thomas (7)

Porta, Alberto (10)

Potse, Mark (12)

Prado, Manuel (6)

Riu, Pere J. (7)

Sabatini, Angelo Maria (6)

Schellart, Nico A. M. (9)

Schmalisch, Gerd (6)

Segers, Patrick (9)

Siebes, Maria (7)

Soltysinski, Tomasz (6)

Song, Charlie (12)

Spaan, Jos A. E. (48)

Stefanovska, Aneta (9)

Steinhoff, Uwe (8)

Streekstra, Geert (7)

Tai, C. (8)

Tyberg, John (7)

van Dam, Peter (6)

van den Wijngaard, Jeroen P. H. M. (8)

Van gemert, Martin (10)

van Haaren, Paul (11)

van Oosterom, Adriaan (8)

VanBavel, Ed (40)

Veltink, Peter (7)

Venema, Henk (6)

Viceconti, Marco (6)

Voss, Andreas (7)

Wahle, Andreas (10)

Westerhof, Berend E. (32)

Westerhof, Nico (32)

Wårdell, Karin (8)

Übeyli, Elif Derya (7)

Zaza, Antonio (6)
Table 2 All 559 reviewers having delivered one or more reports for MBEC in 2012

Abdullah, Haslaile (1)

Abdullah, H. (1)

Abibullaev, Berdakh (1)

Adam, Clayton (1)

Adam, Dan (1)

Akar, Fadi (1)

Akhtar, Muhammad Tahir (1)

Akpinar, Meltem (1)

Al-Ani, Tarik (1)

Al-Naami, Bassam (1)

Al-Nabulsi, Jamal I. (1)

Alkan, Ahmet (1)

Almeida, Vânia (1)

Alomari, Raja S. (1)

Alonso-Valerdi, Luz Maria (1)

Alvarez, Diego (1)

Ambrósio, Paulo Eduardo (1)

Andrade, Adriano O. (1)

Anghel, Traian M. (1)

Anglin, Carolyn (1)

Antelis, Javier M. (1)

Antolini, Renzo (2)

Apolinario, Antonio Lopes (1)

Arfanakis, Konstantinos (1)

Argenti, Fabrizio (1)

Argoud, Fernanda I. M. (1)

Arias-Londono, J. D. (1)

Arslan, Yunus Ziya (2)

Arts, Theo (1)

Arvieux, Catherine (1)

Audenaert, Emmanuel (3)

Avolio, Alberto (3)

Aydin, Serap (1)

Azar, Ahmad Taher (1)

Azevedo-Coste, Christine (1)

Babbs, Charles (1)

Bae, S. H. (1)

Bagnoli, Paola (1)

Baik, Ku Youn (1)

Bakker, Erik (1)

Balbinot, Alexandre (1)

Ballerini, Lucia (1)

Banerjee, Rupak (1)

Barbenel, Joseph (1)

Barbieri, Riccardo (1)

Baritz, Mihaela (2)

Barr, Roger (1)

Barro-Ameneiro, Senen (1)

Basak, Kausik (1) 
Table 2 continued

Baumert, Mathias (2)

Baumgarten, Daniel (1)

Beaumont, Jacques (1)

Bechara, Bernard (1)

Belchior, jadson Claudio (1)

Bera, Tushar Kanti (1)

Bergamini, Elena (1)

Bernabei, Michel (1)

Bernus, O. (1)

Berry, S. L. (1)

Bezerianos, Anastasios (1)

Bezzo, Fabrizio (1)

Bianchi, Anna Maria (1)

Bießmann, Felix (1)

Bigelow, Kimberley (1)

Bignardi, Cristina (1)

Bijak, Manfred (1)

Blanco, Susana (2)

Bo, Antonio Padilha Lanari (1)

Bolander, R. (1)

Bonmassar, Giorgio (1)

Boukens, Bas (1)

Bragin, D. E. (1)

Brill, A. I. (1)

Broekhuizen, B. D. (1)

Brown, Matthew R. G. (1)

Bruijninckx, Cornelis M. A. (1)

Brunner, Clemens (1)

Bunyak, Filiz (1)

Butson, Christopher R. (1)

Caan, M. (1)

Caltun, Ovidiu Florin (1)

Campbell, Paul (1)

Campo, Oscar (1)

Canton, Gador (3)

Cappello, Angelo (1)

Cardinale, Francesco (1)

Cartiaux, Olivier (1)

Casaleggio, Aldo (1)

Castells, Francisco (1)

Cerutti, Sergio (1)

Chamekh, Abdessalem (1)

Chang, Chia-Chi (1)

Chang, Walter H. (1)

Cheliout-Heraut, Fawzia (1)

Chen, Terrence (1)

Chen, Wenxi (2)

Chen, Xiao Bing (1)

Chen, Xin (1)

Chen, Jia-Jin (1)
Table 2 continued

Cherng, Shen (1)

Chien, Jen-Chien (3)

Ching, C. T. (1)

Cho, Sung-Pil (1)

Chow, K. W. (1)

Christensen, Jabba (1)

Chui, Cheekong (1)

Cifrek, Mario (2)

Ciocca, Leonardo (1)

Claiborne, Thomas (3)

Climent, Andreu M. (4)

Colak, Omer H. (1)

Comani, Silvia (1)

Constantinou, C. E. (1)

Contreras-Lamus, Victor (1)

Coronel, Ruben (1)

Cosío, Fernando Arámbula (1)

Cretual, Armel (1)

Crevecoeur, Frédéric (1)

Crevecoeur, Guillaume (1)

Crisco, Joseph J. (1)

Crowe, John (1)

Cunningham, Jimmy (1)

Cuschieri, A. (1)

Cutti, Andrea Giovanni (3)

Cysarz, D. (1)

Dahl, Jeremy J. (1)

Dankelman, Jenny (1)

Dannhauer, M. (1)

Dauwels, Justin (1)

Davalos, Rafael (1)

de Bakker, Jacques (1)

De Carli, Fabrizio (1)

de Cheveigné, Alain (1)

de Groot, Joris (1)

de Melis, Mirko (1)

de Witte, Pieter Bas (1)

Deguchi, S. (1)

Delhaas, Tammo (1)

Deng, S. (1)

Deng, Xiaoyan (1)

Di, Zhao (1)

Diaz, Inaki (1)

Dideriksen, Jakob (2)

Dimitrov, George (1)

Diong, Bill (1)

Dobbe, Johannes G (2)

Doblare, Manuel (1)

Doessel, Olaf (3)

Donaldson, Nicholas de Neufville (1) 
Table 2 continued

Dosen, Strahinja (1)

Dotsinsky, Ivan Assenov (1)

Druzgalski, Chris (2)

Dubini, Gabriele (1)

Dumas, Raphael (1)

Durka, Piotr (1)

Eby, Wayne Martin (1)

Elias, John J. (1)

Englehart, Kevin Brian (1)

Eramian, Mark (1)

Escudero, Javier (1)

Esteban, Francisco (1)

Extramiana, Fabrice (1)

Fancello, Eduardo Alberto (1)

Farina, Dario (1)

Felici, Francesco (1)

Fenster, Aaron (1)

Ferrario, Manuela (2)

Ferri, Massimo (1)

Fevens, Thomas (1)

Figueroa, C. Alberto (2)

Fisher, Lee E. (1)

Folland, Ross (2)

Fontecave-Jallon, Julie (1)

Formaggio, Emanuela (1)

Forman, Jason (1)

Forner-Cordero, Arturo (2)

Fortuna, Luigi (1)

Fougner, Anders (1)

Franz, Thomas (1)

Freivalds, Talivaldis (1)

Fripp, Jurgen (1)

Galbusera, Fabio (1)

Gale, J. T. (1)

Gallasch, Eugen (1)

Gallo, Diego (1)

Galosi, Andrea B. (1)

Gao, Junfeng (1)

Garcia, Fernando (1)

Garcia, G. J. (1)

Gefen, Amit (1)

Georgakis, Apostolos (1)

Geris, Liesbet (1)

Geršak, Gregor (1)

Ghoraani, Behnaz (1)

Giardini, Mario Ettore (1)

Gilbert, Richard (1)

Giraldo, Beatriz (1)

Gleason, Rudolph L. (1)

Godinez-Garcia, Maria (1)
Table 2 continued

Gonçalves, Hernâni (2)

Grebe, Reinhard (1)

Griffith, Boyce (1)

Grimmer, Martin (1)

Groves, David (1)

Guerrero-Mosquera, C. (1)

Guiraud, David (1)

Guo, Yanhui (1)

Gutekunst, David (1)

Hahne, Janne (1)

Haiat, Guillaume (1)

Hall, Charles (1)

Hallez, Hans (1)

Hanson, Ben (1)

Harreby, Kristian Rauhe (1)

Hayano, J. (1)

Heerwaarden, Ronald (1)

Hernández-Hernández, Hugo (1)

Heys, Jeffrey J. (1)

Hirsch, Sven (1)

Hirschfeld, G. (1)

Holden, Arun (1)

Holland, Alex (1)

Holobar, Ales (2)

Hong, K. S. (1)

Horssen van, Pepijn (1)

Hose, Rod (1)

Houben, A. C. H. (1)

Hsieh, Wan-Hsin (1)

Hsu, Wei-Yen (1)

Huang, Haw-Ming (2)

Huang, Ying-zu (1)

Huebner, Thomas (1)

Huiskamp, Geertjan (1)

Hund, Thomas (2)

Hussain, Mozammil (3)

Huxley, Virginia (1)

Hyland, Brian (1)

Iacoviello, Daniela (1)

Infantolino, Benjamin W. (1)

Ishijima, Masa (1)

Rok Istenič (2)

Iwamoto, M. (1)

Jabłoński Ireneusz (1)

Jacquement, Vincent (1)

Jager, Franc (1)

Jankowski, S. (1)

Janusek, Dariusz (1)

Jazbinsek, Vojko (1)

Jiang, Ning (1) 
Table 2 continued

Jimenez-Gonzalez, Aida (2)

Karlen, Walter (1)

Karlik, Bekir (1)

Kato, Tomoko S (1)

Kaufman, Jonathan (1)

Kearney, Robert E. (1)

Keil, F. J. (1)

Kemp, Bastiaan (1)

Kerckhoffs, Roy (1)

Khir, Ashraf William (1)

Kierkegaard, Axel (1)

Kim, Yoon Hyuk (1)

Kim, Youngho (1)

Kim, Tae-Seong (1)

Kimberley, Barry (1)

Knyazev, G. G. (1)

Kochina, Marina (2)

Koivumäki, Janne E. M. (1)

Konings, Maurits K. (1)

Kormendi, Janos (1)

Krams, Rob (1)

Kreuz, Thomas (1)

Krishnan, R. (1)

Krusienski, Dean (2)

Kuijpers, Nico (1)

Kutch, Jason (1)

Kwok, Yeeli (3)

Laguna, Pablo (1)

Lanatà, Antonio (1)

Langlois, Neil (1)

Lanir, Yoram (1)

Larrabide, Ignacio (1)

Lauer, Richard T. (1)

Law, Laura Frey (1)

Leask, Richard L. (1)

Leder, Steven (1)

Lee, Kyoung-Mi (1)

Lethaus, Bernd (1)

Levin, Nathan (1)

Lewis, G. (1)

Li, Zheng (1)

Li, Shutao (1)

Li, Zhi-Yong (2)

Li, X. (1)

Li, Yan (1)

Li, Guanglin (2)

$\mathrm{Li}$, Yaqin (1)

Li, Q. (1)

Liberati, Diego E. I. (1)

Lim, Chwee Teck (1)
Table 2 continued

Lima, Eric (1)

Lin, Sam (1)

Lingala, S. G. (1)

Lithgow, Brian (1)

Liu, Hongbin (1)

Liu, Tao (1)

Liu, Fei (1)

Long, Jinyi (1)

Lopata, Richard (1)

lopez, antonio (1)

Lopomo, Nicola (1)

Lopour, B. A. (1)

Lorrain, Thomas (1)

Lujan, J. Luis (2)

Luo, Xiaoyu (1)

Ma, Jun (1)

Macellari, Velio (1)

Madeiro, João P. V. (1)

Madeleine, Pascal (2)

Maggi, Luis Eduardo (1)

Magjarevic, Ratko (1)

Maia, Jose Everardo B. (1)

Mali, Barbara (1)

Manzanares, Alonso Ramirez (1)

Mao, Haojie (1)

Marais, Patrick (1)

Marateb, Hamid Reza (1)

Marcos, J. Víctor (1)

Mariani, Benoit (1)

Mariani, Sara (1)

Marin, Antonio (1)

Martin, H. D. (1)

Martinez-Mendez, R. (1)

Mason, David Glen (1)

Matsopoulos, George K. (3)

McAdams, Eric (1)

McCane, Brendan (1)

McGill, Kevin (4)

McGrath, Denise (1)

Mehta, Daryush (1)

Meijborg, Veronique (1)

Meineke, Ingolf (1)

Melchionna, Simone (1)

Melillo, Paolo (1)

Melo, Pedro Lopes de (1)

Mendez, Martin Oswaldo (2)

Meraz, Erika G. (1)

Meredith, D. J. (2)

Meriaudeau, Fabrice (1)

Merkus, Daphne (1) 
Table 2 continued

Merletti, Roberto (1)

Mesin, Luca (1)

Micó, Pau (2)

Minh, T. D. (1)

Mitrea, Bogdan (1)

Molinari, Filippo (4)

Moore, James E. (1)

Morbiducci, Umberto (1)

Morega, Alexandru (1)

Moreno, Juan (1)

Morgan, Dennis (1)

Mougiakakou, Stavroula (1)

Moussavi, Zahra (2)

Mulder, Eduard (1)

Munih, Marko (1)

Murase, T. (1)

Muresan, Leila (1)

Muskulus, Michael (1)

$\mathrm{Na}$, Yang (1)

Nagura, Takeo (1)

Nazarpour, Kianoush (1)

Neagu, Daniel (1)

Nederveen, A. (1)

NG, David (1)

Niazi, Imran Khan (1)

Nicolaou, Nicoletta (1)

Niederer, Steven (1)

Niizeki, Kyuichi (1)

Nikkhah, M. (1)

Nilsson, Lena (1)

Nivala, Michael (1)

Noshiro, Makoto (2)

Novo, Jorge (1)

Oh, Duck-won (1)

Oomens, Cees W. J. (1)

Oostendorp, Thom (1)

Oosterhoff, Peter (1)

Orizio, Claudio (1)

Oskoei, Mohammadreza Asghari (1)

Pandian, S. P. (1)

Panerai, Ronney (1)

Pantall, Annette (1)

Papaharilaou, Yannis (2)

Parsaei, Hossein (1)

Patil, Mothiram Keelanje (1)

Pauling, John (1)

Pekkan, Kerem (2)

Penzel, Thomas (3)

Petrone, Nicola (1)

Phlypo, Ronald (1)
Table 2 continued

Pichler, Gerhard (1)

Piella, Gemma (1)

Pinzon-Morales, Ruben Dario (1)

Poh, Ming-Zher (1)

Polak, Adam G. (1)

Pollard, Andrew Emil (1)

Popovic, Dejan (1)

Porcaro, Camillo (1)

Porta, Alberto (1)

Potse, Mark (3)

Prado, Manuel (1)

Punit, Prakash (1)

Quaresma, Claudia (1)

Ramachandram, Dhanesh (1)

Raspopovic, Stanisa (1)

Rattay, Frank (1)

Rauch, Lukasz (1)

Raul, Jean Sebastien (1)

Renner, Jochen (1)

Requena Carrión, Jesús (1)

Reulen, Jos P. H. (1)

Rieta, Jose Joaquin (1)

Rincón Vallejos, Francisco Javier (1)

Roan, Esra (1)

Rodriguez, Javier (3)

Rodriguez Merzagora, A. (1)

Roduit, Charles (1)

Rosanova, Mario (1)

Rotenberg, Alexander (1)

Royston, Thomas (1)

Rugonyi, Sandra (1)

Sabater, Jose Maria (1)

Sadat, Umar (1)

Sadleir, R. (1)

Saeys, Yvan (1)

Saggio, Giovanni (1)

Sahakian, Alan (1)

Salsac, Anne-Virginie (1)

Sansone, Mario (1)

Sawacha, Zimi (1)

Schefler, Bernard (1)

Schellart, Nico A. M. (1)

Schmalisch, Gerd (1)

Schreuder, Martijn (1)

Schubert, Christian (1)

Scoponi, Marco (1)

Secomb, Tim (1)

Segal, R. A. (1)

Segers, Patrick (2)

Sejdic, Ervin (4) 
Table 2 continued

\section{Selvaraj, Nandakumar (1)}

Senhadji, Lotfi (1)

Seppä, Ville-Pekka (2)

Sermesant, Maxime (1)

Severens, M. (1)

Severi, Stefano (1)

Shelley, Kirk (1)

Sheppard, Lawrence (1)

Shipley, Rebecca (2)

Shiralkar, Manish (1)

Siddiqui, Mohammad Khubeb (1)

Siebes, Maria (1)

Silvestri, Sergio (1)

Skallerud, Bjorn (1)

Smolensky, Michael (1)

Sokolis, Dimitrios P. (2)

Sola-Soler, Jordi (1)

Solis-Escalante, Teodoro (1)

Solomon, Jeffrey (1)

Soltysinski, Tomasz (4)

Song, Charlie (1)

Souvenir, Richard (1)

Spaan, Jos A. E. (8)

Spahn, Gunter (1)

Spanakis, Emmanouil G. (1)

Spitzer, Bernhard (1)

Stashuk, Daniel (2)

Stefanovska, Aneta (1)

Steinhoff, Uwe (1)

Stevanella, Marco (1)

Stoitsis, John (1)

Subit, D. (3)

Sugawara, Motoaki (1)

Swain, Michael (1)

Taeymans, Jan (1)

Tai, C. (4)

Takahashi, T. (1)

Tamez-Pena, Jose Gerardo (1)

Tarin, Cristina (1)

Terriault, Patrick (1)

Tew, Garry (1)

Thedens, Daniel (1)

Thomas, Eoin (1)

Thonnard, Jean-Louis (1)

Tiinanen, Suvi (1)

Togawa, Tatsuo (1)

Tran, Yvonne (1)

Tsakalis, Kostas (1)

Tu, Jiyuan Y. (1)

Turner, Scott (1)
Table 2 continued

Umale, Sagar (1)

Ur Rehman, N. (1)

van Dam, Peter (1)

van de Vosse, Frans (1)

van den Wijngaard, Jeroen P. H. M. (2)

van der Meijden, Olivier (1)

Van gemert, Martin (3)

van Houwelingen, Marc Jan (1)

VanBavel, Ed (4)

Vella, Dominic (1)

Veltink, Peter (1)

Ventikos, Yiannis (1)

Vergari, Claudio (1)

Vigmond, Edward (1)

Viik, Jari (1)

Villiger, Michael (1)

Virk, Gurvinder (1)

Virkkala, Jussi (1)

Voisin, Julien (1)

Voss, Andreas (2)

Wagenaar, Daniel (1)

Wahle, Andreas (1)

Wallman, Mikael (1)

Wang, Vincent (1)

Wang, Y. (1)

Ward, Leigh (1)

Webster, John G. (1)

Weeren, René (1)

Wei, Q. (1)

Weiss, Bela (1)

Wenk, Jonathan F. (1)

Westerhof, Berend E. (3)

Westerhof, Nico (7)

Wiklund, Urban (1)

Wilkes, Tony (1)

Witte, Herbert (1)

Wolters, Carsten (1)

Woodbridge, N. (1)

Wårdell, Karin (3)

$\mathrm{Xu}$, G. (1)

$\mathrm{Xu}$, Yun (1)

Yamanishi, Yoshihiro (1)

Yang, Lin (1)

Yang, Feng (1)

Yin, Frank (1)

Yoon, Gilwon (1)

Young, Diana (1)

Yu, Wenwei (1)

Yuenyong, S. (1)

Zajc, M. (1) 
Table 2 continued

Zazula, Damjan (1)

Zhang, Henggui (2)

Zhang, Yongjie (1)

Zhang, Zhiguo (1)

Zhang, Haihong (1)

Zhao, Xuefeng (1)

Zhao, Jichao (1)

Zheng, Guoyan (1)

zlochiver, sharon (2)

Zysset, Philippe (2)

\section{Reference}

1. Spaan JA, Coronel R (2012) Fifty years of publishing in biomedical engineering: reflections after 7 year editorship. Med Biol Eng Comput 50(12) (this issue) 